\title{
ON TIME HISTORY ANALYSIS WITH STEPS LARGER THAN THE STEPS OF EARTHQUAKE RECORDS INDEPENDENT FROM THE FREQUENCY CONTENTS
}

\author{
Aram Soroushian \\ International Institute of Earthquake Engineering and Seismology (IIEES) \\ No. 21, West Arghavan, North Dibajee, S. Lavasani (Farmaniyeh), Tehran 19537, Iran \\ e-mail: a.soroushian@iiees.ac.ir
}

Keywords: Time Integration, Digitized Excitation, Computational Cost, Accuracy, Errors Control, Response Frequency Content.

\begin{abstract}
Time integration is the most versatile tool in analysis of semi-discretized equations of motion. The computational cost might be considerable, especially when the excitation is available as a digitized record. Concentrating on analysis of structural systems against earthquakes, a technique is proposed in 2008 for reducing the analysis computational cost by summarizing the record of the ground motion. The main parameter to be set before implementation of the technique is the largest period of oscillations with considerable contribution in the response. Apparently, the definition of the parameter is vague, and besides we cannot easily estimate the parameter prior to the analysis. In this paper, the computational cost reduction technique is enhanced to a technique that can be simply implemented regardless of the above-mentioned parameter. The way of implementation is modified, such that the need to the parameter is eliminated without adding any new parameter or ambiguity. The basis of the enhancement is the error control comment traditionally recommended in the practice. As observed in numerical tests, by implementing the enhanced technique, besides further clarity, the time integration analyses can be more efficient compared to traditional analyses.
\end{abstract}




\section{INTRODUCTION}

Time integration is a versatile tool in analysis of transient behaviors; see [1-10]. Concentrating on structural analysis against digitized ground motion records, after discretization in space, the mathematical model can be stated as [11-18]:

$$
\begin{aligned}
& \mathbf{M} \ddot{\mathbf{u}}+\mathbf{f}_{\text {int }}=-\mathbf{M} \Gamma \ddot{\mathbf{u}}_{g} \quad 0 \leq t<t_{\text {end }} \\
& \mathbf{u}(t=0)=\mathbf{u}_{0} \\
& \dot{\mathbf{u}}(t=0)=\dot{\mathbf{u}}_{0} \\
& \mathbf{f}_{\text {int }(t=0)=\mathbf{f}_{\text {int }_{0}}} \\
& \mathbf{Q}
\end{aligned}
$$

In Eqs. (1), $\mathbf{M}$ is the mass matrix, $\mathbf{f}_{\text {int }}$ implies the internal force, $\Gamma$ stands for the matrix relating the excited degrees of freedom to the rest of the structure $[17,18], \ddot{\mathbf{u}}_{g}$ denotes the excitation at the excited degrees of freedom, e.g. ground acceleration (including multi-support excitations) [17, 18], $\mathbf{Q}$ represents the restrictions originating in nonlinearity, e.g. see [19, 20], $t$ and $t_{\text {end }}$ respectively represent the time and the time interval, and " 0 " as a right subscript indicates that the argument is at its initial status [17, 18]. The main process of time integration is pictorially addressed in Fig. 1, where it is apparent that the integration step size, $\Delta t$, has the main role in the analysis. The broadly accepted comment for selecting the integration step size is as stated below $[12,17,18,21,22]$ :

$$
\Delta t \leq \cong \operatorname{Min}\left(\frac{T}{\chi}, \Delta t_{c r}, \Delta t_{r}, f_{f} \Delta t\right)
$$

In Eq. (2), $T$ denotes the size of the smallest oscillatory period with considerable contribution in the response, $\Delta t_{c r}$ represents the largest integration step size leading to numerically stable responses, $\Delta t_{r}$ implies the smallest step size, according to which, we accept to have the record of the response, and ${ }_{f} \Delta t$ stands for the step size, by which, the earthquake (or digitized excitation) is recorded (see [21]), and as addressed in [23],

$$
\chi=\left\{\begin{array}{cc}
10 & \text { linear behavior } \\
100 & \text { nonlinear behavior } \\
1000 & \text { nonlinear behavior involved in impact }
\end{array}\right.
$$

Integration station

○

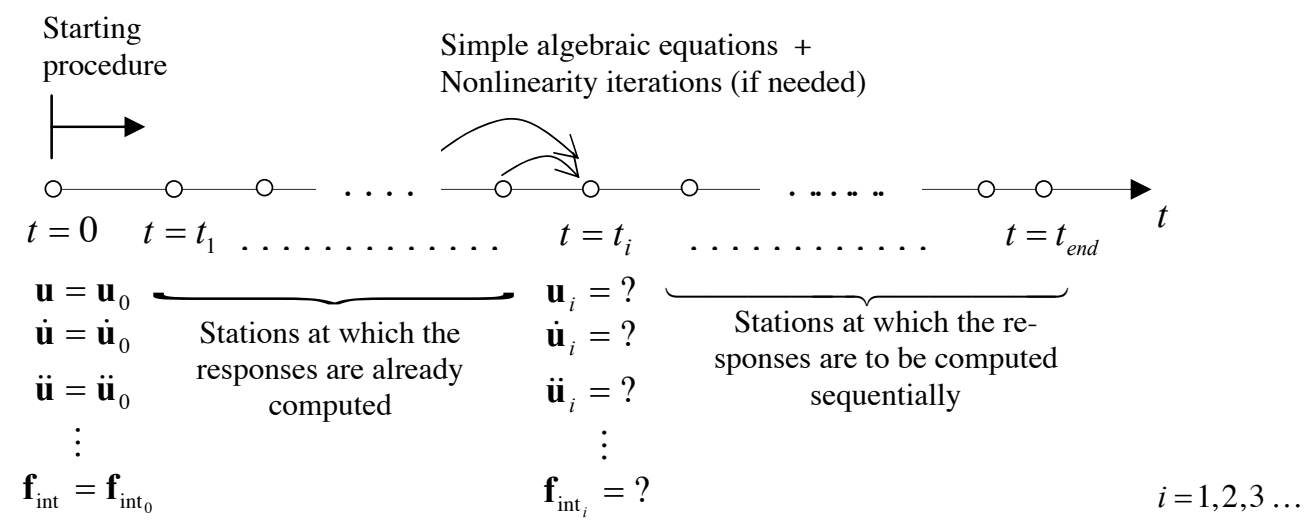

Figure 1: Pictorial description of time integration analysis [21]. 
When the excitation step size, ${ }_{f} \Delta t$, is such small to satisfy

$$
{ }_{f} \Delta t<\operatorname{Min}\left(\frac{T}{\chi}, \Delta t_{c r}, \Delta t_{r}\right)
$$

Eq. (2) may result in values for the integration step size not needed for the accuracy of the time integration and disadvantageous for the computational cost. For these cases, a technique is proposed in 2008 [24], that replaces the original excitation record with a record digitized at the larger steps, ${ }_{f} \Delta t_{n e w}=n_{f} \Delta t \quad\left(n \in Z^{+}-\{1\}\right)$. The original steps, ${ }_{f} \Delta t$, are enlarged by the integer scale $n$, and the record data is changed, such that to preserve responses convergence (see [24]). As the consequence, after the time integration analysis, the response deviates negligibly from the response of the ordinary analysis with steps equal to ${ }_{f} \Delta t$, i.e. the technique leads to less computational cost, in the price of no specific inaccuracy, when [21]

$$
{ }_{f} \Delta t<_{f} \Delta t_{\text {new }} \leq \operatorname{Min}\left(\frac{T}{\chi}, \Delta t_{c r}, \Delta t_{r}\right)
$$

(for detailed explanations, on this technique, see [21, 24, 25], and for other computational cost reduction techniques potentially considering digitized excitations, and the comparison of the techniques, see [21, 26-29]). An enhanced version of the technique [24, 25] takes into account fractional enlargement of step sizes; see [30]. (Table 1 reports some experiences on the technique.) As implied above, the amount of the integration steps enlargement, $n$, is to be set such that $\left(Z^{+}\right.$is the set of positive integer numbers):

$$
\begin{aligned}
& { }_{f} \Delta t_{\text {new }}=n_{f} \Delta t \\
& n_{f} \Delta t \leq \operatorname{Min}\left(\frac{T}{\chi}, \Delta t_{c r}, \Delta t_{r}\right)<(n+1)_{f} \Delta t \quad, \quad n \in Z^{+}-\{1\}
\end{aligned}
$$

\begin{tabular}{lcc}
\hline System analyzed & $\begin{array}{c}\text { Cost reduction with no signifi- } \\
\text { cant additional inaccuracy (\%) }\end{array}$ & Source \\
\hline SDOF system & 75 & {$[25]$} \\
\hline 2-DOF nonlinear system & 49.27 & {$[25]$} \\
\hline Eight storey shear frame & 80 & {$[31]$} \\
\hline Thirty-storey building & 50 & {$[32]$} \\
\hline 3-component earthquakes & 66.7 & {$[33]$} \\
\hline Silos with linear/nonlinear behavior & 77.65 & {$[34,35]$} \\
\hline Water tank (the structure and the fluid) & 66.7 & {$[36,37]$} \\
\hline Building in pounding & 12.7 & {$[38]$} \\
\hline Bridges with linear/nonlinear behavior & $45-80$ & {$[39,40]$} \\
\hline Power stations & $>50$ & {$[41]$} \\
\hline Regular residential buildings & $50-87$ & {$[42]$} \\
\hline Bridges with pre-stressed elements subjected to & $30-70$ & {$[39]$} \\
multi-support excitations & $50-80$ & {$[43]$} \\
\hline Residential building with irregularities in height & $>50$ & {$[44]$} \\
\hline Space Structures & $>50$ & {$[45]$} \\
\hline A cooling tower & $>50$ & {$[46,47]$} \\
\hline Milad telecommunication tower (linear/nonlinear) & $>80$ & {$[49]$} \\
\hline Beams/beams assemblages subjected to digitized static loading & $>50$ & \\
\hline Three real buildings (linear and nonlinear) & & \\
\hline
\end{tabular}

Table 1: Experiences regarding time integration step size enlargement technique proposed in [24]. 
Considering the role of $T$ in Eqs. (5) and (6) and the value of $n$, and the ambiguity in the definition of $T$ [21], the objective in this paper is to eliminate the parameter $T$ from the implementation of the technique proposed in [24, 25]. The main idea can be summarized in: (1) replacing the accuracy-based approach of time integration analysis with a computational-costbased approach [50], (2) consistent and conventional changes of $n$ and ${ }_{f} \Delta t$ in the first relation in Eq. (6), when needed, and with less importance (3) Engineering-based control of the accuracy. The main idea is stated in the next section. The remaining theory leading to a new and practical way for implementation of the technique proposed in [24, 25] are discussed later. The numerical study is presented in continuation, and finally, the paper is concluded with a brief set of the achievements and remarks.

\section{THE MAIN IDEA}

The purpose of Eq. (2) and its predecessor (set for continuous excitations) [24, 25, 30], i.e.

$$
\Delta t \leq \cong \operatorname{Min}\left(\frac{T}{\chi}, \Delta t_{c r}, \Delta t_{r}\right)
$$

and specifically the term $\frac{T}{\chi}$ in this equation is providing sufficient accuracy. This is while, as recommended not only in the structural dynamics literature, e.g. see [17], but also in the structural codes, e.g. see [23], and the literature of numerical solution of differential equations, e.g. [51], the accuracy of time integration analysis is to be controlled after the analysis. The most conventional comment in this regard is repetition of the analysis with half steps and somehow comparing the responses [17, 23]; for other practical comments, for instance, see [52, 53]. In other words, traditionally, after the time integration analysis, we would rather check the accuracy by somehow comparing the responses with some other responses supposed to be more accurate. In brief, we once adapt the analysis to the accuracy prior to the analysis, via $\frac{T}{\chi}$ in Eqs. (2) or (7), and once again control the accuracy after the analysis by repetition of the analysis. Since the first control is involved in ambiguities [21], it is reasonable to decrease the accuracy controlling role of Eqs. (2) and (7) by eliminating the term $\frac{T}{\chi}$. This would replace Eqs. (2), (5), and (6), with the much simpler equations below:

$$
\begin{aligned}
& \Delta t \leq \cong \operatorname{Min}\left(\Delta t_{c r}, \Delta t_{r}\right) \\
& \Delta t=n_{f} \Delta t={ }_{f} \Delta t_{\text {new }} \\
& { }_{f} \Delta t<_{f} \Delta t_{n e w} \leq \operatorname{Min}\left(\Delta t_{c r}, \Delta t_{r}\right) \\
& n_{f} \Delta t \leq \operatorname{Min}\left(\Delta t_{c r}, \Delta t_{r}\right)<(n+1)_{f} \Delta t \quad, \quad n \in Z^{+}-\{1\}
\end{aligned}
$$

with attention to which, after implementing the technique proposed in [24, 25] while

$$
n \leq \cong \frac{\operatorname{Min}\left(\Delta t_{c r}, \Delta t_{r}\right)}{{ }_{f} \Delta t}
$$

we can carry out the analysis. Therefore, the enlargement scale $n$ can be determined independent from $T$, prior to implementation of the technique and carrying out the analysis. Meanwhile, in view of an existing comment $[13,54,55]$, on numerical stability, i.e.

$$
\Delta t_{c r} \rightarrow \infty
$$


In view of Eq. (13) and the fact that the technique proposed in [24, 25] is enhanced to a technique taking into account fractional values for $n$ [30], Eq. (12) can simply be replaced with:

$$
n:=\frac{\Delta t_{r}}{{ }_{f} \Delta t}
$$

leading to more simplicity and likely less computational cost for the time integration. The accuracy still needs investigation.

\section{CONTROL OF ACCURACY}

The discussion presented in the previous section provides the capability to implement the technique proposed in [24, 25] or its enhanced version proposed in [30], independent from $T$ (and in fact independent from the frequency content of the response) and with steps sized below:

$$
\begin{gathered}
\Delta t=n_{f} \Delta t \quad\left(=\Delta t_{r}\right) \\
n=\frac{\Delta t_{r}}{{ }_{f} \Delta t}
\end{gathered}
$$

Regarding repetition of the analysis with half or smaller steps, questions exist. They are; which term(s) in the right hand side of the first relation in Eq. (15) and how much should each term be decreased in the repetition? In response, in view of the necessity of errors' consistent decrease discussed in $[24,56]$, in order to preserve the second order convergence (see also [13, 24, 54-56]), $n$ and ${ }_{f} \Delta t$ would rather converge consistently. In this regard, by considering the first analysis and its repetition carried out respectively with steps below:

$$
\begin{aligned}
\Delta t_{1} & =n_{1}{ }_{f} \Delta t_{1} \\
\Delta t_{2} & =n_{2}{ }_{f} \Delta t_{2}
\end{aligned}
$$

it is sufficient to satisfy (pay attention to the fact that the ideal limiting values of $n$ and ${ }_{f} \Delta t$ are respectively ' 1 ' and ' 0 '):

$$
\begin{aligned}
& \frac{n_{1}-1}{2}=n_{2}-1 \\
& \frac{{ }_{f} \Delta t_{1}}{2}={ }_{f} \Delta t_{2}
\end{aligned}
$$

leading to

$$
\begin{aligned}
& n_{2}=\frac{n_{1}+1}{2} \\
& f^{\Delta t_{2}}=\frac{{ }_{f} \Delta t_{1}}{2}
\end{aligned}
$$

Accordingly and in view of the fact that

$$
n_{i}>1 \quad, \quad i=1,2
$$

the analysis and error control will converge (never exactly correspond) to the ordinary control of errors, based on repetition with half steps. 
Consequently, after we enlarge the excitation steps from ${ }_{f} \Delta t$ to $\Delta t_{r}$, and carry out the first analysis, considering ${ }_{f} \Delta t_{1}={ }_{f} \Delta t$ and $n_{1}=\frac{\Delta t_{r}}{{ }_{f} \Delta t}$ (see Eq. (14)),

$$
\Delta t_{1}=\frac{\Delta t_{r}}{{ }_{f} \Delta t} f \Delta t=\Delta t_{r}
$$

we can repeat the analysis with the smaller steps below:

$$
\begin{aligned}
& \Delta t_{2}=\frac{n_{1}+1}{2} \frac{f \Delta t_{1}}{2} \\
& n_{1}=\frac{\Delta t_{r}}{{ }_{f} \Delta t}
\end{aligned}
$$

and study the accuracy by comparing the responses obtained from the two analyses, and if needed carry out the second and more repetitions, by step sizes obtained from

$$
\begin{aligned}
& n_{j}=\frac{n_{j-1}+1}{2} \\
& f_{f}^{\Delta t_{j}}=\frac{{ }_{f} \Delta t_{j-1}}{2} \\
& \Delta t_{j}=n_{j}{ }_{f} \Delta t_{j}
\end{aligned}
$$

For the sake of clarity, it is essential to note that Eqs. (22) imply that in each repetition of the analysis the excitation steps are to be scaled down to ${ }_{f} \Delta t_{j}$ and the excitation digitized at the smaller steps should be determined using linear interpolation. Then the excitation record to be implemented in the analysis can be obtained, by applying the technique proposed in [30] to the record digitized ${ }_{f} \Delta t_{j}$, taking into account the corresponding value of $n$, addressed in Eqs. (22), i.e. $n=n_{j}$.

And finally, the criterion to control the accuracy can be set arbitrarily considering different norms [57]. Nevertheless, as a selection consistent with practice, the comment of the seismic code of New Zealand [23] is taken into account. Accordingly, the relative difference of the peak target responses in an analysis and its repetition is to be not more than 5\%. However, since different from the comment stated in [23], the step sizes do not precisely halve in each repetition (see Eqs. (16) and (17), the peaks difference need to be controlled as noted below [58] ( $P_{1}$ and $P_{2}$ are the peaks, i.e. maximum absolute values, of the target response throughout the time interval respectively in the an analysis and its repetition):

$$
\frac{\left|P_{1}-P_{2}\right|}{P_{2}} \leq \frac{0.05}{3}\left[\left(\frac{2 n_{1}}{n_{2}}\right)^{2}-1\right]
$$

\section{NUMERICAL STUDY}

Since the discussion in the previous section is carried out in a mostly rigorous manner and meanwhile this paper reports an "in progress" study (in its final stages), only one example is presented here. (Four examples from experiences addressed in Table 1 are being tested on the performance of the approach proposed in this paper; see [58].)

Consider the structural system defined in Fig. 2 and Table 2, where the lateral displacement of the top floor is the target response (with the exact top floor displacement displayed in Fig. 3 and the exact peak value equal to $0.177058778 \mathrm{~m}$ ), the average acceleration method of 
A Typical Shear Frame
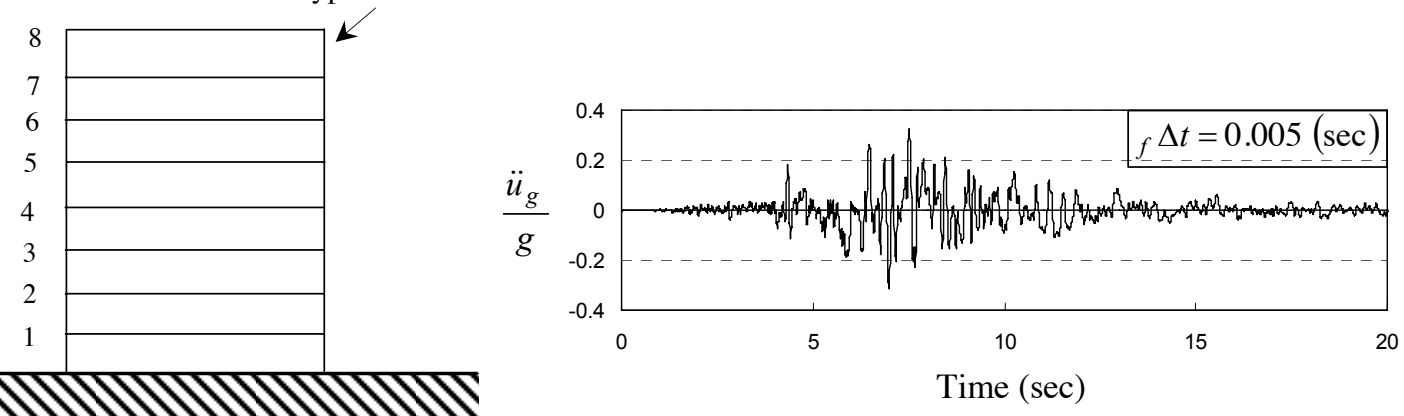

Figure 2: Structural system under consideration: (a) Structural model, (b) Ground acceleration.

\begin{tabular}{|c|c|c|c|}
\hline Storey & Mass (Kg) & Stiffness $\left(\mathrm{N} / \mathrm{m}^{2}\right)$ & $\begin{array}{l}\text { Damping } \\
(\mathrm{N} / \mathrm{m} / \mathrm{sec})\end{array}$ \\
\hline 1 & 1036E4 & 860E7 & \multirow{8}{*}{0} \\
\hline 2 & 1034E4 & 840E7 & \\
\hline 3 & 1032E4 & $820 \mathrm{E} 7$ & \\
\hline 4 & 1030E4 & 700E7 & \\
\hline 5 & $1028 \mathrm{E} 4$ & 680E7 & \\
\hline 6 & 1026E4 & 660E7 & \\
\hline 7 & 1024E4 & 640E7 & \\
\hline 8 & 1022E4 & $620 \mathrm{E} 7$ & \\
\hline
\end{tabular}

Table 2: Some properties of the structural system in Fig. 2.

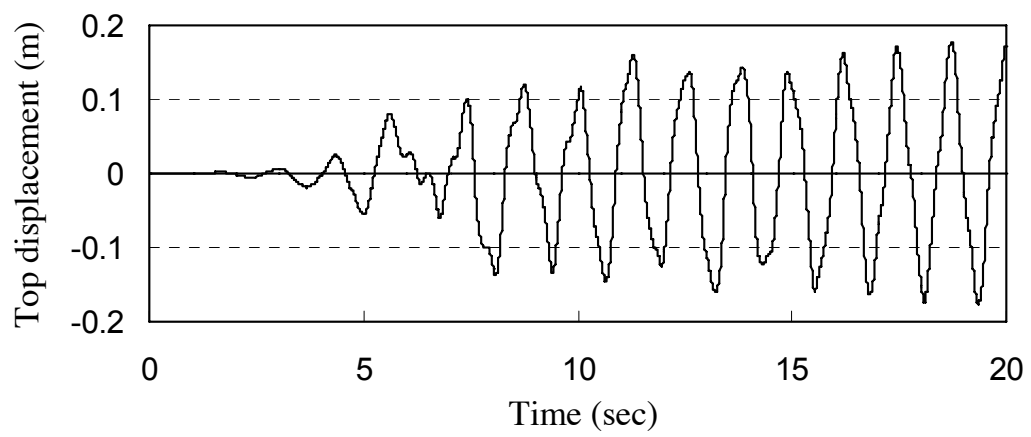

Figure 3 - Exact history of the top displacement.

Newmark $[17,18,59]$ is the integration method, and $\Delta t_{r}=0.05 \mathrm{sec}$.

In implementation of the traditional analysis approach, we first time integrate with steps equal to $\Delta t_{1}={ }_{f} \Delta t=0.005 \mathrm{sec}$, and then repeat the analysis with steps equal to $\Delta t_{2}=0.5 \Delta t_{1}=0.0025 \mathrm{sec}$, and if the accuracy control is not passed, we sequentially repeat the analysis with half steps, till the relative difference of the peak top floor displacements in two sequential analyses is not more than $5 \%$. This condition is satisfied after the first repetition (the peaks in the first two analyses equal $0.176781 \mathrm{~m}$ and $0.176975 \mathrm{~m}$, apparently in less than $5 \%$ relative difference), implying the total number of integration steps below:

$$
N_{T_{1}}=\frac{20}{0.005}+\frac{20}{0.5 \times 0.005}=12000
$$


In implementation of the way of implementation (of the technique proposed in [24, 25]) proposed in this paper, the peaks difference satisfies the condition, which is now changed to Eq. (23), again after two sequential analyses (the two peaks respectively equal to $0.1848861 \mathrm{~m}$ and $0.17566966 \mathrm{~m})$, carried out with steps stated below:

$$
\begin{aligned}
& \Delta t_{1}=\Delta t_{r}=0.05=10 \times 0.005 \quad\left({ }_{f} \Delta t_{1}={ }_{f} \Delta t=0.005 \mathrm{sec}\right) \\
& \Delta t_{2}=\frac{10+1}{2} \times 0.0025=0.01375 \quad\left({ }_{f} \Delta t_{2}=0.5_{f} \Delta t_{1}=0.0025 \mathrm{sec}\right)
\end{aligned}
$$

and the condition is satisfied as stated below:

$$
\begin{aligned}
& \frac{|0.184866-0.175670|}{0.175670} \leq \frac{0.05}{3}\left[\left(\frac{20}{5.5}\right)^{2}-1\right] \\
& 0.0523 \leq 0.20372
\end{aligned}
$$

The corresponding total number of integration steps is reported below:

$$
N_{T_{2}}=\frac{20}{0.05}+\frac{20}{0.01375}=1856
$$

in view of Eq. (24), implying about $85 \%$ reduction of total number of integration steps compared to the traditional approach (in view of the linear behavior, the computational cost has undergone a similar decrease; see $[21,24,60]$ ), and even in consideration of the third analysis only for the new approach, still about $54 \%$ reduction of cost is provided. The target responses both very accurate are pictorially not recognizable and hence are not included here for the sake of brevity. Consequently, not only the new way of implementation of the technique is clearer than its predecessor, but also at least for this example the analysis is more efficient. The study is being continued with other integration methods, other excitations, other structural systems, other values of $\Delta t_{r}$, and while also considering different nonlinear behaviors; see [58].

\section{CONCLUDING REMARKS}

After addressing a practical ambiguity in implementation of a time integration computational cost reduction technique (also see [21]), a new way of implementation is proposed, totally eliminating the weak point. Specifically and as the consequences,

1. For simpler efficient time integration analysis against ground motions, the computational cost reduction technique proposed in 2008 [24], and enhanced in 2017 [30], is implemented in a completely different way, considering less accuracy-controlling role in the integration step size selection stage.

2. Compared to its predecessor [24, 25], the new way of implementation is much simpler and clearer, in consistence with the engineering accuracy-controlling comments/conventions, and still can be more efficient compared to ordinary time integration analyses.

Finally, it is worth noting that since the earthquake data recording instrumentation is in rapid progress [61] and consideration of records with smaller steps is recommended [62], the need to the technique proposed in $[24,25]$, and the related researches will increase. Accordingly more research on the continuation of the discussions presented in this paper is strongly recommended. 


\section{REFERENCES}

[1] Z. Zlatev, R. Berkowicz, L.P. Prahm, Implementation of a variable step size variable formula method in the time integration part of a code for treatment of long-range transport of air pollutants. Journal of Computational Physics, 55, 278-301, 1984.

[2] A. Bonelli, O.S. Bursi, Generalized- $\alpha$ methods for seismic structural testing. Earthquake Engineering and Structural Dynamics, 33, 1067-1102, 2004.

[3] D.M. Doolin, N. Sitar, Time integration in discontinuous deformation analysis. Journal of Engineering Mechanics ASCE, 130, 249-257, 2004.

[4] O.S. Bursi, L. He, A. Bonelli, P. Pegon, Novel generalized- $\alpha$ methods for interfiled parallel integration of heterogeneous structural dynamic systems. Journal of Computational and Applied Mathematics, 234, 2250-2258, 2010.

[5] G. da Costa Villar, J.C. Menezes, M.V. Donadon, Accuracy and numerical stability of direct time integration schemes applied to rotordynamics. $12^{\text {th }}$ Pan-American Congress of Applied Mechanics (PACAM XII), Port of Spain, Trinidad, January 2-6, 2012.

[6] A. Akbarzadeh-Sharbaf, D.D. Giannacopoulos, Finite-element time-domain solution of the vector wave equation in doubly dispersive media using Möbius transformation technique. IEEE Transactions on Antenna and Propagation, 61, 4158-4166, 2013.

[7] L.T. Yun, L. QuingBin, Z. ChongBin, An efficient time-integration method for nonlinear dynamic analysis of solids and structures. Science China Physics, Mechanics, \& Astronomy, 56, 798-804, 2013.

[8] B.A. Erickson, J. Nordstrom, Stable, high order accurate adaptive schemes for long time, highly intermittent geophysics problems. Journal of Computational and Applied Mathematics, 271, 328-338, 2014.

[9] X.W. Tang, X.W. Zhang, R. Uzuoka, Novel Adaptive Time Stepping Method and Its Application to Soil Seismic Liquefaction Analysis. Soil Dynamics and Earthquake Engineering, 71, 100-113, 2015.

[10] K. Kpodzo, L. Fourment, P. Lasne, P. Montmitonnet, An accurate time integration scheme for arbitrary rotation motion: application to metal forming formulation. International Journal of Material Forming, 9, 71-84, 2016.

[11] H. Kardestuncer, Finite element handbook. McGraw Hill, 1987.

[12] K.J. Bathe, Finite element procedures. Prentice-Hall, 1996.

[13] T. Belytschko, T.J.R. Hughes, Computational methods for transient analysis. Elsevier, 1983.

[14] T. Belytschko, W.K. Liu, B. Moran, Non-linear finite elements for continua and structures. John Wiley \& Sons, 2000.

[15] A. Soroushian, New methods to maintain responses' convergence and control responses' errors in the analysis of nonlinear dynamic models of structural systems, $\mathrm{PhD}$ Dissertation. University of Tehran, 2003. (In Persian)

[16] J. Henrych, Finite models and methods of dynamics in structures. Elsevier, 1990.

[17] R.W. Clough, J. Penzien, Dynamics of structures. McGraw-Hill, 1993.

[18] A.K. Chopra, Dynamics of structures: theory and application to earthquake engineering. Prentice-Hall, 1995.

[19] P. Wriggers, Computational contact mechanics. Wiley, 2002. 
[20] T.J.R. Hughes, K.S. Pister, R.L. Taylor, Implicit-explicit finite elements in nonlinear transient analysis. Computer Methods in Applied Mechanics and Engineering, 17, 159$182,1979$.

[21] A. Soroushian, Integration step size and its adequate selection in analysis of structural systems against earthquakes. In Eds. M. Papadrakakis, V. Plevris, N.D. Lagaros, eds., Computational Methods in Earthquake Engineering Vol 3, Springer, 2017.

[22] J.F. McNamara, Solution schemes for problems of nonlinear structural dynamics. Journal of Pressure Vessel Technology ASME, 96, 147-155, 1974.

[23] Structural Design Actions Part 5: Earthquake Actions-New Zealand Commentary (Supplement to NZS 1170.5:2004) (2004) NZS 1170.5 Supp 1. Standards New Zealand, 2004.

[24] A. Soroushian, A technique for time integration with steps larger than the excitation steps. Communications in Numerical Methods in Engineering, 24, 2087-2111, 2008.

[25] A. Soroushian, Direct time integration with steps larger than the steps by which the excitations are digitized, Report 7510. International Institute of Earthquake Engineering and Seismology (IIEES), 2011. (In Persian)

[26] S.Y. Chang, Integrated equations of motion for direct integration methods. Structural Engineering and Mechanics, 13, 569-589, 2002.

[27] M. Hosseini, I. Mirzaei, Simplification of earthquake accelerograms for rapid time history analysis based on the impulsive load concept. $10^{\text {th }}$ World Congress on Computational Mechanics (WCCM X), Sao Paulo, Brazil, July 8-13, 2012.

[28] A. Soroushian, Time integration with step sizes less depending on the steps of excitation: I. SDOF systems, Report 7509-P89-1. International Institute of Earthquake Engineering and Seismology (IIEES), 2009. (In Persian)

[29] A. Saaed, A technique for faster seismic analysis of MDOF structural systems, M.sc. Dissertation. International Institute of Earthquake Engineering and Seismology (IIEES), 2012. (In Persian)

[30] A. Soroushian, Y.Zarabimanesh, K. Soleymani, S.M. Khalkhali, A new technique for fractional enlargement of integration steps in transient analysis against digitized excitations. International Conference on Structural Engineering Dynamics (ICEDyn 2017), Ericeira, Portugal, July 3-5, 2017.

[31] A. Soroushian, On practical performance of a technique recently proposed for time integration analysis with less computational cost. $17^{\text {th }}$ International Congress on Sound \& Vibration (ICSV17), Cairo, Egypt, July 18-22, 2010.

[32] A.Soroushian, A. Aziminejad, A more efficient seismic analysis of tall buildings by implementing a recently proposed technique. $6^{\text {th }}$ International Conference Seismology and Earthquake Engineering (SEE6), Tehran, Iran, May 16-18, 2011.

[33] O. Bahar, S. Ramezani, Faster time integration analysis for building structures subjected to 3-component earthquakes. $3^{\text {rd }}$ ECCOMAS Thematic Conference on Computational Methods in Structural Dynamics and Earthquake Engineering (COMDYN 2011), Island of Corfu, Greece, May 26-28, 2011.

[34] F. Nateghi, M. Yakhchalian, On less computational costs for analysis of silos seismic behaviors by time integration. $3^{\text {rd }}$ ECCOMAS Thematic Conference on Computational Methods in Structural Dynamics and Earthquake Engineering (COMPDYN 2011), Island of Corfu, Greece, May 26-28, 2011. 
[35] F. Nateghi, M. Yakhchalian, An investigation into the effectiveness of a technique proposed for reducing computational cost of time integration in the analysis of silos seismic behaviors. $11^{\text {th }}$ US National Congress on Computational Mechanics (USNCCM 2011), Minneapolis, USA, July 25-28, 2011.

[36] A. Soroushian, A. Saaed, M. Arghavani, M. Rajabi, M.M. Sharifpour, Less computational costs in the analysis of reservoirs seismic behaviours by time integration. $10^{\text {th }} \mathrm{Bi}$ ennial Conference on Vibration Problems (ICoVP-2011), Prague, Czech Republic, September 5-8, 2011.

[37] A. Soroushian, P. Farshadmanesh, S. Azad, On the essentiality of techniques to enlarge analysis steps in time integration against digitized excitations. International Journal for Earthquake Engineering and Seismology (JSEE), 17, 43-60, 2015.

[38] A. Soroushian, On the performance of a recent technique for more efficient time integration in severe seismic conditions. $1^{\text {st }}$ World Congress on Advances in Structural Engineering and Mechanics (ASEM'11), Seoul, South Korea, September 18-22, 2011.

[39] S. Azad, A study on accelerating time history analysis of bridges, M.Sc. Dissertation. International Institute of Earthquake Engineering and Seismology (IIEES), 2015. (In Persian)

[40] A. Soroushian, A. Vasseghi, M. Hosseini, On practical performance of a technique for more efficient dynamic analysis in view of real seismic analysis of a bridge structural system. In M. Papadrakakis, M. Fragiadakis, V. Plevris, eds., Computational Methods in Earthquake Engineering Vol 3, Springer, 2013.

[41] M. Bastami, A technique for more efficient time integration applied to seismic analysis of power substation equipment. Proceedings of the $11^{\text {th }}$ World Congress on Computational Mechanics (WCCM XI), Barcelona, Spain, July 20-25, 2014.

[42] A. Sabzei, On the performance of a recent technique for seismic analyses computational cost reduction when applied to buildings structural systems, M.Sc. Dissertation. International Institute of Earthquake Engineering and Seismology (IIEES), 2013. (In Persian)

[43] A.A. Hadad, Reducing computational costs in time integration analyses of buildings with irregularities in height because of mass, M.Sc. Dissertation. International Institute of Earthquake Engineering and Seismology (IIEES), 2015. (In Persian)

[44] A. Garakaninezhad, R.K. Moghadas, On the performance of a technique to accelerate time integration when applied to space structures analyses. $5^{\text {th }}$ ECCOMAS Thematic Conference on Computational Methods in Structural Dynamics and Earthquake Engineering (COMPDYN 2013), Kos Island, Greece, June 12-14, 2013.

[45] A. Soroushian, A. Jahani Mehrnoosh, Y. Zarabimanesh, M.H. Ghondaghsaz, A. Baiani, A. Zakizade, On the performance of a computational cost reduction technique when applied to cooling towers transient analysis. $7^{\text {th }}$ European Congress on Computational Methods in Applied Sciences and Engineering (ECCOMAS VII), Crete Island, Greece, June 5-10, 2016.

[46] A. Soroushian, S.R. Mirghaderi., Y.Zarabimanesh, A. Jahani Mehrnoosh, A. Zakizadeh, M.H. Ghondaghsaz, On the possibility to accelerate time history analysis of the Milad telecommunication tower. 10 ${ }^{\text {th }}$ National Congress on Civil Engineering (ICCE 10), Tehran, Iran, April 19-20, 2017. 
[47] Y.Zarabimanesh, On the possibility to reduce the computational cost of time history analysis of the Milad telecommunication tower, M.sc. Dissertation. International Institute of Earthquake Engineering and Seismology (IIEES), 2017. (In Persian)

[48] E.M. Farahani, A. Ganji, S. Maalek, A. Soroushian, Reduction of computational cost in finite element analysis of beams against digitized static loadings. $10^{\text {th }}$ National Congress on Civil Engineering (ICCE 10), Tehran, Iran, April 19-20, 2017.

[49] H. Ghondaghsaz, A study on a recent technique for more efficient seismic analysis applied to concrete and steel buildings, M.Sc. Dissertation. International Institute of Earthquake Engineering and Seismology (IIEES), in Progress. (In Persian)

[50] A. Soroushian, A new approach for practical time integration analysis. $6^{\text {th }}$ ECCOMAS Thematic Conference on Computational Methods in Structural Dynamics and Earthquake Engineering (COMPDYN 2013), Rhodes Island, Greece, June 15-17, 2017.

[51] E. Hairer, G. Wanner, Solving ordinary differential equations II: Stiff and differentialalgebraic problems. Springer, 1996.

[52] A. Soroushian, P. Wriggers, J. Farjoodi, Asymptotic upper bounds for the errors of Richardson Extrapolation with practical application in approximate computations. International Journal for Numerical Methods in Engineering, 80, 565-595, 2009.

[53] P. Ruge, A priori error estimation with adaptive time-stepping. Communications in Numerical Methods in Engineering, 15, 479-491, 1999.

[54] T.J.R. Hughes, The finite element method: linear static and dynamic finite element analysis. Prentice-Hall, 1987.

[55] W.L. Wood, Practical time stepping schemes. Oxford, 1990.

[56] S.N. Penry, W.L. Wood, Comparison of some single-step methods for the numerical solution of the structural dynamic equation. International Journal for Numerical Methods in Engineering, 21, 1941-1955, 1985.

[57] B. Noble, J.W. Daniel, Applied linear algebra. Prentice Hall, 1977.

[58] A. Soroushian, Direct time integration from earthquake induced equations of motion with steps larger than conventional and least dependence to the frequency content of the response, Report 7537. International Institute of Earthquake Engineering and Seismology (IIEES), in progress. (In Persian)

[59] N.M. Newmark, A Method of Computation for Structural Dynamics. Journal of Engineering Mechanics ASCE, 85, 67-94, 1959.

[60] D.M. Monro, FORTAN 77. Edward Arnold, 1987.

[61] J. Havskov, G. Alguacil, Instrumentation in earthquake seismology (modern approaches in geophysics). Springer, 2004.

[62] W. Du, C.L. Ning, Influence of time step of ground motions on site effect and structural response analyses for long duration earthquakes. Journal of Earthquake Engineering (just accepted). 